\title{
Principal Component Analysis of Multi-Pigment Scenario in Full-Color Electrophoretic Display
}

\author{
Yen-Hsing Lu and Chung-Hao Tien
}

\begin{abstract}
In this paper, we present a methodology to characterize the colorimetric performance of a full-color electrophoretic display (EPD) with multiple pigment formation. We use the principal component analysis to formulate the composite hybrid colormixing system by using a single eigen-spectral component for each sub-pixel channel. Proposed model was validated to predict the chromatic features with high accuracy $\left(\Delta \mathrm{E}_{\text {AVE_CIEDE20oo }}<\right.$ 0.2 ) for both micro-cup type and micro-encapsulated type EPD samples. This study is effective to uncover the underlying physical features from only colorant-mixture information in a novel color system.
\end{abstract}

Index Terms-Full-color electrophoretic display (EPD), principal component analysis, colorant-mixture.

\section{INTRODUCTION}

$\mathbf{T}$ ECHNOLOGIES in reflective display (or also known as electronic-paper, e-Paper) have made a rapid progress in the last decade. E-Paper is a direct view electronic display that is either bi-stable or nearly bi-stable with the benefit of extremely low power consumption. Generally, there are several scenarios to implement the bi-stability (or nearly bi-stability), including electrophoretic [1]-[7], electrofluidic [8], [9], bi-stable liquid crystal display (LCD) [10], [11], electrowetting [12], [13] and other emerging approaches [14]. Despite jeopardized by the great success of emissive displays and their accompanying PC devices, e-Paper still has potential to leverage its strongest advantages which emissive counterparts are hard to achieve, such as superior energy saving, fine contrast under sunlight, continuous roll-to-roll manufacturing, flexible format, or even paper-like reading comfort. One major goal of such non-self-luminous media is to retain many of the desirable characteristics of color printed paper, while keeping ubiquitous communication via different electronic interfaces. The unique feature containing both hard copy rendering and soft copy operation has drawn enormous attention to the public, thus drive many studies to pursue more suitable modes and manufacturing methods.

Electrophoretic display (EPD) is one of the remarkable approaches among e-Paper family, due to its successful technologies such as micro-encapsulated type from E Ink, micro-cup type from SiPix in monochromatic, independently movable col-

Manuscript received December 04, 2012; revised March 20, 2013; accepted April 23, 2013. Date of publication May 13, 2013; date of current version October 09,2013 . This work was supported by the National Science Council of Taiwan under Contract NSC 99-2221-E-009-067-MY3.

The authors are with the Department of Photonics, National Chiao Tung University, Hsinchu 30010, Taiwan (e-mail: chtien@mail.nctu.edu.tw).

Color versions of one or more of the figures are available online at http:// ieeexplore.ieee.org.

Digital Object Identifier 10.1109/JDT.2013.2260527

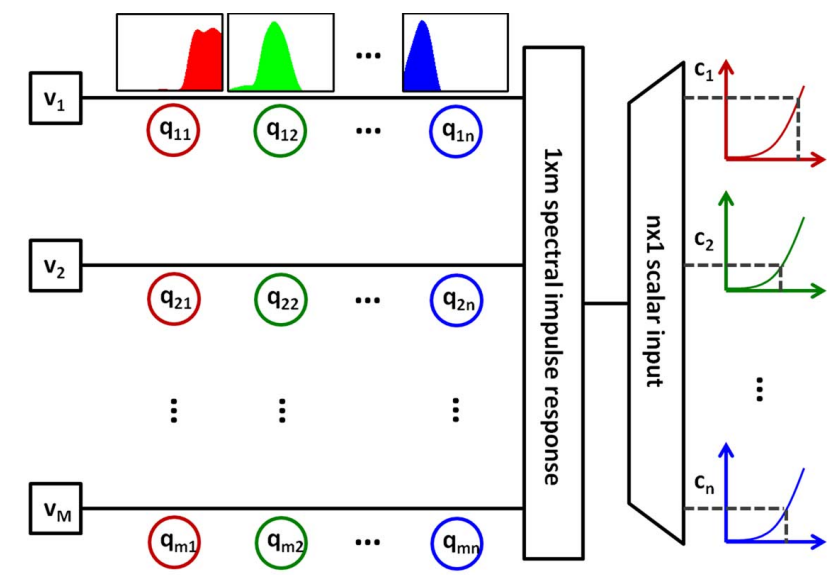

$\left.\begin{array}{c}\text { Resulatant } \\ \text { spectrum } \\ v_{1} \\ v_{2} \\ \vdots \\ v_{m}\end{array}\right]=\left[\begin{array}{cccc}q_{11} & q_{12} & \cdots & q_{1 n} \\ q_{21} & q_{22} & \cdots & q_{2 n} \\ \vdots & \vdots & \ddots & \vdots \\ q_{m 1} & q_{m 1} & \cdots & q_{m n}\end{array}\right] \bullet \bullet\left[\begin{array}{c}c_{1} \\ c_{2} \\ \vdots \\ c_{n}\end{array}\right]$

Fig. 1. The colorimetric characterization performs the matrix-vector multiplication of $\mathbf{v}=\mathbf{Q c}$. System transfer matrix $(\mathbf{Q})$ is composed of $n$ featuring spectral channels determining the significantly spectral components in color system.

ored particles (IMCP) type from Fuji-Xerox [7], respectively. Electrophoresis is the motion of particles dispersed in solution or medium under the influence of an applied electric field in either vertical or horizontal direction. In terms of display or electronic printing, the particles are embedded in thin-film layers. The color and gray level of the display can be determined by the reflective properties of the ink particles, or of a surface behind the fluid or their combination. The ink particles can move either in the plane of the panel or perpendicular to it in response to the applied field. Taking electrophoretic system as a case, if e-Paper can achieve adequate colored images, we are wondering what is the underlying physical feature from only colorant-mixture information? Since the amazing similarity with conventional print technology, by which the multicolor images are produced by placing small dots of ink with a specific spectral reflectance side-by-side, we are asked if there exists a unifying scheme to describe the nature of multi-pigment transposition in e-Paper, and if so, whether we can characterize it in a systematic way borrowed from well-established color print technology?

To help guide our investigation, a linear transformation between device-dependent and colorimetric representations is introduced at first [15]. Fig. 1 illustrates the transformation mechanism that executes a matrix-vector multiplication. Without loss 


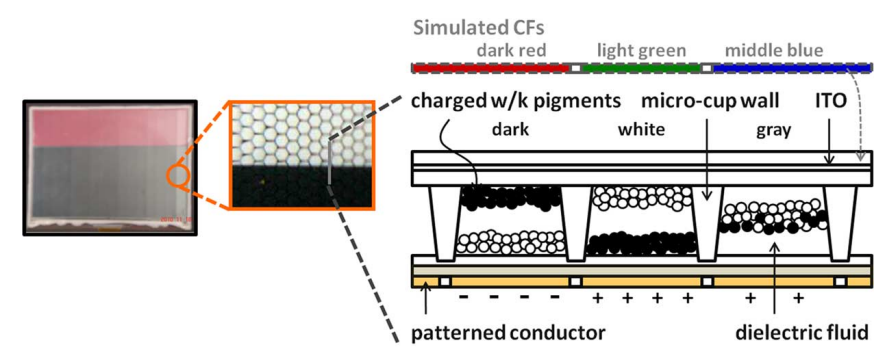

(a)
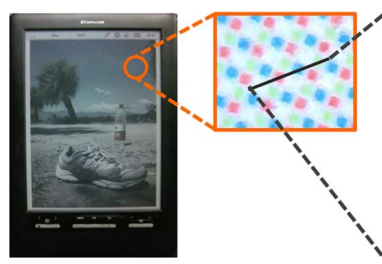

charged w/k pigments oil

CF ITO
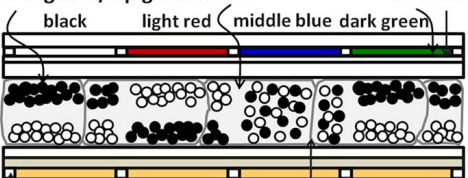

-+++
patterned conductor

(b)

Fig. 2. Schematic diagram of (a) monochromatic micro-cup and (b) full-color micro-encapsulated electrophoretic display. A net of electric fields are applied across the cells to control the distributions of the charged multi-pigments. The spectral absorptance of dielectric fluid or oil, which is subject to the position of the multi-pigments, offers the sub-pixel the resulting lightness. Moreover, the EPD achieves full-color approach with the pixel layout of simulated RGB in (a) and practical RGBW in (b), respectively. Different amounts of absorbed light from sub-pixels are introduced into our eyes and mixed additively on retina accordingly. Therefore, the color-mixing principle of the full-color EPD is a complex system which cannot be easily characterized by simple additive or subtractive color mixing theorem.

of generality, the medium is assumed to be characterized by $n$ featuring spectral channels (e.g., for conventional emissive display, $n=3$, the matrix represents the spectral superposition of red/green/blue channels) sampled by $m$ wavelength points to form an $m \times n$ linear transformation matrix $(\mathbf{Q})$. The system characteristic matrix determines which spectral components are significant in a color system. The $n$-by- 1 vector $\left(\mathbf{c}_{\mathbf{i}}\right)$ offers a set of scalar ratios, subject to input digital counts, to determine the intensity of individual channels. The resulted spectrum $(\mathbf{v})$ can be obtained via Q-c multiplication accordingly.

In terms of EPD, its colorimetric performance is mainly determined by single (or multiple) pigment transposition. A pigment is a colorant that is usually not soluble in the medium in which it is applied and incorporated into the thin film in particle form. Pixel technology involves the combination of pigment (single or multiple), dielectric fluid (monochrome or transparent), filter, and electrode layout. Fig. 2(b) illustrates a vertical colorant transposition with simplified micro-encapsulated architecture, whose pixel arrangement is a two-colorant mixing with RGBW filter. In this two particles system, the charged black or white pigments move perpendicular to the plane of panel, and the reflectance of each subpixel is determined by whether the particles are at the front or back.

In addition to the gray modulation by colorant transposition, color EPD shall equip red, green, and blue color filters to allow full-color rendering. Based on micro-cup architecture, SiPix also developed an alternative paradigm to create a full-color system called dual mode switching. In this approach, white colorant particles are embedded in red, green or blue colored dyes and a black layer of light absorbing material is placed behind each sub-pixel. The electric fields are arranged so that the particles can move sideways as well as forward and back. According to Heikenfeld's study, bi-primary mixing is very suited for colorant-transposition technologies. This is an advantage, because colorant transposition technologies exhibit some of the highest reflectance and color fraction [16]. Except for color filter and colored dyes approaches, Fuji-Xerox introduced a novel color EPD using independently movable colored particles with different threshold fields [7]. The technique disperses second primary colored particles in identical cell to enable truly printing-like e-Paper. No matter what mixing scheme a color system use, obviously it is not possible to model such composite mixing scheme by a pure subtractive or additive theory. Instead, numerical regression and interpolation are the most practical approaches to characterize such complex color mechanism, but at an expense of huge measured efforts [17].

Principal component analysis, abbreviated PCA, has been widely used as a mathematical tool in color technology [18]-[20]. One major function of PCA is to find the principal directions that a set of data oriented. The major and minor axes imply that many physical significance hidden by a large amount of end-to-end measurement data. In this paper, we aim to employ PCA to uncover the underlying physical principles related to a complex color-mixing scheme. In case of EPD with composite mixing via micro-cup and micro-encapsulated structure, we expect to precisely reconstruct color model of a full-color EPD while extracting primary spectral features for multi-pigments transposition. The method is kind of learning-based reconstruction, i.e., a preliminary measured data set is used to build up the color model. In order to reduce the superfluous information, the spectral reflectances of the EPD samples are merely estimated from eigen-spectral components by using PCA and projection matrix. Color difference between sample spectra and their spectral estimation for a set of samples will be discussed. Finally, we will examine the proposed PCA-based methodology by two case studies (micro-cup type and micro-encapsulated type color EPD).

\section{Methodology}

PCA is well described by means of vector-matrix language. All the scientific symbols and abbreviations utilized in following section are listed in Table I where scalars are represented by italic characters. Vectors and matrices are denoted by bold-faced lower-case and upper-case letters, respectively. Here we merely make a quick review of the PCA in color technology. The complete matrix algebra will not be reviewed here, as detailed information can be found in many classical publications [21]-[23].

First of all, we analyze a variance-covariance or correlation structure of a spectral reflectance set in matrix form. As we described in Fig. 1, the column vectors of matrix constitute a vector space. For conventional liquid crystal displays, the column vectors are mainly determined by the spectral transmittance of the color filter. But those in EPD become more complicated because the spectral vectors arise from composite color mixing combination, including colorant-mixture, fluid, filter, or microstructure. Hence we must use a skill to simplify the matrix and uncover the spectral properties of each component channel from a set of measured data. 
TABLE I

NOMENCLATURE

\begin{tabular}{|c|c|c|c|}
\hline \multicolumn{4}{|c|}{ Abbreviations } \\
\hline $\mathbf{A}$ & $\begin{array}{l}\text { variance-covariance } \\
\text { matrix }\end{array}$ & $\mathbf{M}$ & $\begin{array}{l}\text { color matching function } \\
\text { matrix }\end{array}$ \\
\hline $\mathbf{Q}$ & $\begin{array}{l}\text { orthonormal } \\
\text { eigenvector matrix }\end{array}$ & $\mathbf{R}$ & projection matrix \\
\hline $\mathbf{T}$ & tristimulus matrix & $\Lambda$ & eigenvalue matrix \\
\hline c & scalar ratio vector & $\mathbf{q}$ & eigenvector \\
\hline t & tristimuls vector & $\overline{\mathbf{t}}$ & mean tristimulus vector \\
\hline $\mathbf{v}$ & sample vector & $\overline{\mathbf{v}}$ & mean vector \\
\hline$c$ & scalar & $n$ & numbers of samples \\
\hline$m$ & $\begin{array}{l}\text { numbers of } \\
\text { sampled wavelength }\end{array}$ & $m^{\prime}$ & $\begin{array}{l}\text { numbers of } \\
\text { principal components }\end{array}$ \\
\hline$q$ & sample number & $\lambda$ & eigenvalue \\
\hline$X Y Z$ & tristimulus value & $\bar{X} \bar{Y} \bar{Z}$ & mean tristimulus value \\
\hline \multicolumn{4}{|c|}{ Superscripts and subscripts } \\
\hline i & $\mathrm{i}^{\text {th }}$ sample & $\mathrm{T}$ & transpose operator \\
\hline $\mathrm{PC}$ & principal component & & \\
\hline
\end{tabular}

Given a spectral reflectance set, $\mathbf{V}$, an $m \times n$ matrix with $m$ sampled wavelengths and $n$ number of colors (e.g., spectral reflectance of color-ramp shown by EPD), denoted as a set of column vectors of $\left[\mathbf{v}_{\mathbf{1}} \mathbf{v}_{\mathbf{2}} \ldots \mathbf{v}_{\mathbf{n}}\right]$. As each sample color $\mathbf{v}_{\mathbf{i}}$, is measured within the visible spectrum between $400 \mathrm{~nm}$ and $700 \mathrm{~nm}$ at $10-\mathrm{nm}$ intervals; $\mathbf{v}_{\mathbf{i}}$ is a vector of 31 components. Similarly, as we have a set of 216 sample colors that EPD can achieve, the dimension of this characteristic matrix becomes $(m \times n)=(31 \times 216)$.

A mean vector, $\overline{\mathbf{v}}$, is the average of the entire sample set. The physical significance of the mean spectrum is the colorant neutral (like the DC bias of a learning database) of an EPD sample.

$$
\overline{\mathbf{v}}=\frac{1}{n} \sum_{i=1}^{n} \mathbf{v}_{\mathbf{i}} .
$$

The difference between the spectral reflectance and mean spectrum $\left(\mathbf{v}_{\mathbf{i}}-\mathbf{v}\right)$ presents the spectral variance. As we correlate the variance among $n$ spectral reflectance entry, a variance-covariance matrix (A) can be obtained as following:

$$
\mathbf{A}=\frac{1}{n-1} \sum_{i=1}^{n}\left(\mathbf{v}_{\mathbf{i}}-\overline{\mathbf{v}}\right)\left(\mathbf{v}_{\mathbf{i}}-\overline{\mathbf{v}}\right)^{T}
$$

where $T$ is the matrix-vector transpose operator. Notice that $m$ wavelengths confine the sample set in an $m$-dimensional vector space. Each entry of $\mathbf{A}, a_{i j}$, describes the data correlation between $i$ th and $j$ th spectral channel where $i$ and $j \leq n$. The higher absolute value of the term in $\mathbf{A}$ is in response to the higher correlation between two spectral features. It also implies that when $a_{i j}$ is zero, the $i$ th and $j$ th spectral features are totally uncorrelated. Since $\mathbf{A}$ is a symmetric matrix based on the formation in (2), variance-covariance matrix can be factored into a perfect form (orthogonal)(diagonal)(orthogonal $)^{\mathrm{T}}$ by change of basis [24]:

$$
\mathbf{A}=\mathbf{Q} \Lambda \mathbf{Q}^{\mathbf{T}}
$$

where $\mathbf{Q}$ is an $m$-by- $m$ matrix constituted by a set of orthonormal eigenvectors $\left(\mathbf{q}_{1}, \mathbf{q}_{2}, \mathbf{q}_{\mathbf{3}} \ldots \mathbf{q}_{\boldsymbol{m}}\right)$ of matrix $\mathbf{A}$. The eigenvectors are linearly independent and indentify the principal directions that a set of reflectance spectra oriented. $\boldsymbol{\Lambda}$, a diagonal matrix, is the eigenvalues of the corresponding eigenvectors. If we descend the diagonal terms $\left(\lambda_{1}, \lambda_{2}, \ldots, \lambda_{m}\right)$ of $\Lambda$, the $\lambda_{1}$ reveals the most dominant variance along the new spectral axis (i.e., eigenvector $\mathbf{q}_{1}$ ). The new axes (or bases) represent the directions with maximum variance and effectively reduce the scale of matrix. The total percentage variance defined by the first $m^{\prime}$ eigenvectors is shown as

$$
\text { Percent Variance }=100 \times \frac{\sum_{i=1}^{\boldsymbol{m}^{\prime}} \lambda_{i}}{\sum_{\boldsymbol{i}=1}^{\boldsymbol{m}} \lambda_{i}} .
$$

Equation (4) can be utilized as an assessment to estimate the dimensionality of the given sample set. For example, if the first $m^{\prime}$ eigenvectors represent $99 \%$ of total variance $\left(m^{\prime} \leq m\right)$, then the original matrix $\mathbf{A}$ can be well approximated as a downsize form:

$$
\begin{aligned}
\mathbf{A} & \cong\left[\begin{array}{llll}
\mathbf{q}_{1} & \mathbf{q}_{2} & \cdots & \mathbf{q}_{\mathbf{m}^{\prime}}
\end{array}\right]\left[\begin{array}{llll}
\lambda_{1} & & & \\
& \lambda_{2} & & \\
& & \ddots & \\
& & & \lambda_{m^{\prime}}
\end{array}\right]\left[\begin{array}{c}
\mathbf{q}_{1} \\
\mathbf{q}_{2} \\
\vdots \\
\mathbf{q}_{\mathbf{m}^{\prime}}
\end{array}\right] \\
& =\mathbf{Q}_{\mathbf{p c}} \boldsymbol{\Lambda}_{\mathbf{p c}} \mathbf{Q}_{\mathbf{p c}}^{\mathbf{T}}
\end{aligned}
$$

where the subscript, pc, stands for principal component. Comparing (5) with (3), the dimensionality is reduced from $m$ column vectors to $m^{\prime}$ primary eigenvectors if the given sample set is re-distributed in a new eigenvector coordinate system.

To achieve the linear transformation, we introduce a projection matrix, $\mathbf{R}$, which is an orthogonal projector, calculated from principal eigenvector matrix, $\mathbf{Q}_{\mathbf{p c}}$ :

$$
\mathbf{R}=\mathbf{Q}_{\mathrm{pc}}\left(\mathbf{Q}_{\mathrm{pc}}^{\mathbf{T}} \mathbf{Q}_{\mathrm{pc}}\right)^{-1} \mathbf{Q}_{\mathrm{pc}}^{\mathrm{T}}=\mathbf{Q}_{\mathrm{pc}} \mathbf{Q}_{\mathrm{pc}}^{\mathrm{T}} .
$$

Accordingly, the sample value, $\mathbf{v}_{\mathbf{i}}$, can be approximately reconstructed by mean vector, $\overline{\mathbf{v}}$, and $\mathbf{R}-\mathbf{v}$ multiplication as follows:

$$
\mathbf{v}_{\mathbf{i}} \cong \overline{\mathbf{v}}+\mathbf{R} \mathbf{v}_{\mathbf{i}}=\overline{\mathbf{v}}+\mathbf{Q}_{\mathbf{p c}} \mathbf{c}_{\mathbf{i}}
$$

where $\mathbf{c}_{\mathbf{i}}$ is the scalar ratio vector whose entries indicate the projection along eigenvector direction. Now, (7) is further expressed with matrix extension:

$$
\left[\begin{array}{c}
v_{1} \\
v_{2} \\
\vdots \\
v_{m}
\end{array}\right]_{\boldsymbol{i}}=\left[\begin{array}{c}
\bar{v}_{1} \\
\bar{v}_{2} \\
\vdots \\
\bar{v}_{m}
\end{array}\right]+\left[\begin{array}{cccc}
q_{11} & q_{12} & \cdots & q_{1 m^{\prime}} \\
q_{21} & q_{22} & \cdots & q_{2 m^{\prime}} \\
\vdots & \vdots & \ddots & \vdots \\
q_{m 1} & q_{m 2} & \cdots & q_{m m^{\prime}}
\end{array}\right]_{\mathbf{p c}}\left[\begin{array}{c}
c_{1} \\
c_{2} \\
\vdots \\
c_{m^{\prime}}
\end{array}\right]_{\boldsymbol{i}}
$$

Recall the linear transformation employed to describe the rendering mechanism of a display system in Fig. 1, each column vector in the principal component matrix $\left(\mathbf{Q}_{\mathbf{p c}}\right)$ from the reduction of the eigenvector matrix of the variance-covariance matrix (A) can be regarded as the characteristic vector analysis of EPD. Until now, the physical features of the multi-pigment mixture for an EPD have been revealed by the PCA-based method. However, the spectral formation is not convenient for color reproduction due to the redundant dimension [25].

In the perspective of color communication among multi-media, employing simplified quantification such as 

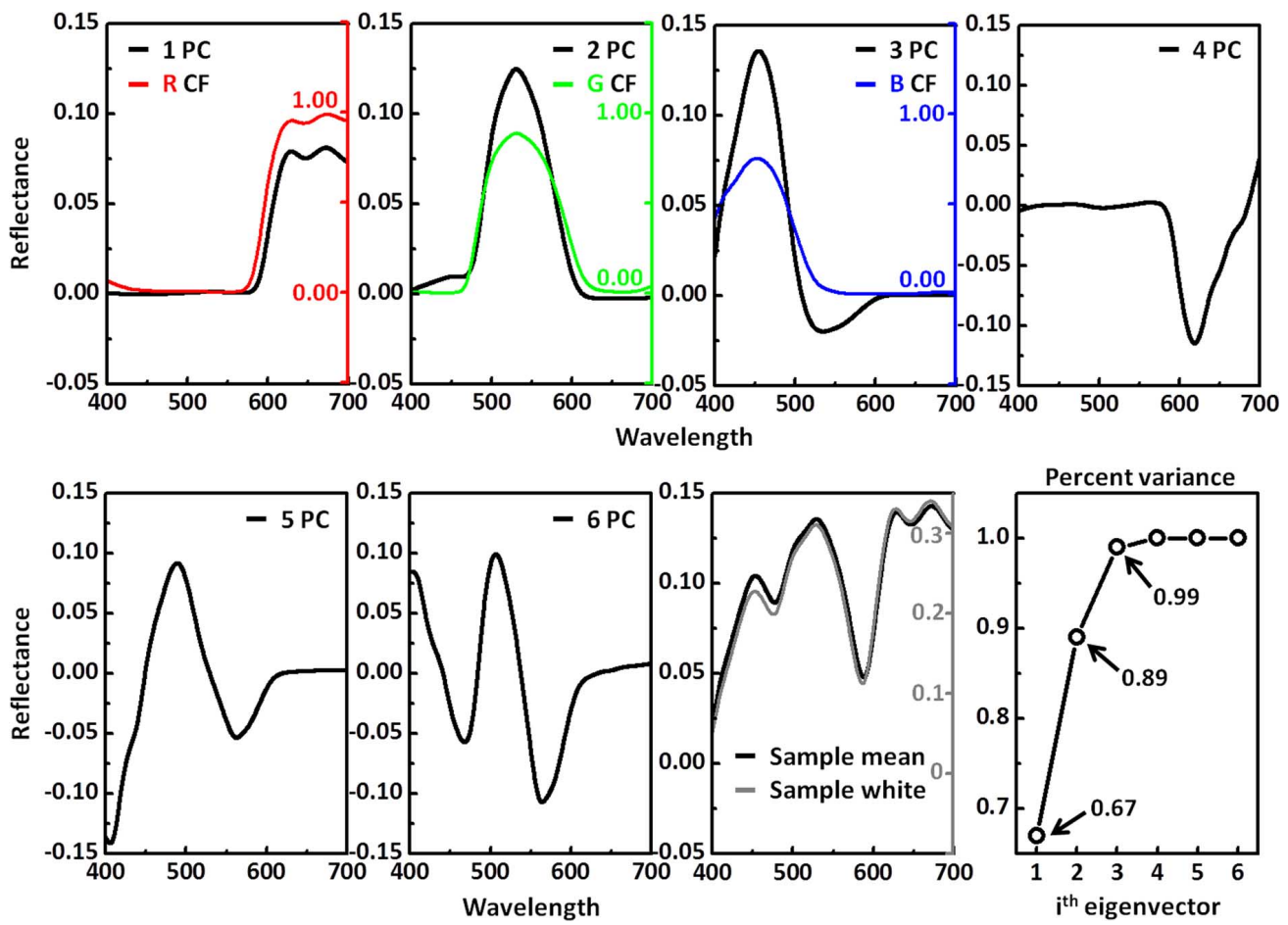

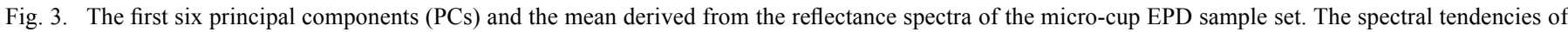

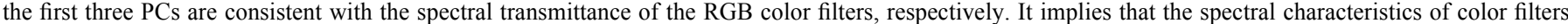
strongly dominant the significant directions in this case study.

tristimulus values or CIELAB values is more intuitive and more feasible. Therefore, turning (7) into a low dimension form containing three entries of each column vector is necessary for practicality. An $m$-by-3 matrix, $\mathbf{M}$, where the three columns are color-matching functions is introduced to reduce dimension:

$$
\mathbf{M}^{\mathbf{T}} \mathbf{v}_{\mathbf{i}}=\mathbf{M}^{\mathbf{T}} \overline{\mathbf{v}}+\mathbf{M}^{\mathbf{T}} \mathbf{Q}_{\mathbf{p c}} \mathbf{c}_{\mathbf{i}}
$$

The $m$-dimensional column vectors from (7) are now projected into three-dimensional human-visual-system subspace and transformed to the corresponding projection:

$$
\mathbf{t}_{\mathbf{i}}=\overline{\mathbf{t}}+\mathbf{T}_{\mathbf{p c}} \mathbf{c}_{\mathbf{i}}
$$

where $\mathbf{t}$ is the column vector of tristimulus values, and the 3-by- $m^{\prime}$ matrix, $\mathbf{T}_{\mathbf{p c}}$, uncovers the colorimetric features of the system characteristic matrix. We can see that the systematic description of the colorant-mixture for a multi-pigments EPD is from $m$-dimensional spectral formation to three-dimensional colorimetric formation while the scalar vectors are identical in both equations. This colorimetric expression will benefit further applications such as evaluating model accuracy and communicating information across different electronic interfaces.

\section{CASES STUDY}

In order to validate the characterizing technique, we adopt two full-color e-Papers, micro-cup EPD and micro-encapsulated EPD, to be our case studies. In measurement setup, the TOPCON ${ }$ SR-UL1R spectroradiometer was used to measure the colorimetric information shown on the EPD. It features the working luminance range of $0.005-3000 \mathrm{~cd} / \mathrm{m}^{2}$ with the reliable accuracy of $\pm 2 \%$ and \pm 0.002 in luminance and chromaticity, respectively. The $45^{\circ}: 0^{\circ}$ setup, the SR-UL1R measured from the normal side of the EPD and the light source illuminated from the $45^{\circ}$ position, was adopted. In addition, the spectra reflectance of each color sample was measured for $2^{\circ}$ observer under CIE illuminant F7. All the color data had been measured five times and then averaged in terms of accuracy and stability.

\section{A. Micro-Cup Type Color EPD}

Since the dual mode switching sample involving RGB colored dyes was not commercially available, a 9-inch monochromatic micro-cup (MC) EPD sample combined with simulated RGB color filters was examined as the first case and shown in Fig. 2(a). Apart from the essential structures such as polymer micro-cup and transparent fluid, the EPD included white and black pigments with opposite polarity to enhance the intrinsic contrast ratio. Based on the simplest pixel layout, commercial RGB color filters (CFs) were placed side-by-side over the top of micro-cup. The well-known spectral transmittances of the CFs were employed to calculate the performance. Each individual RGB channel was capable of 16 substantial gray levels to accomplish 4096 colors in total. The fundamental measurement of a set of 216 sampling grid colors created by 6 digit counts of red, green, and blue was displayed over the MC EPD as the learning database for principal component analysis.

The results of the principal directions changed from the set of sample pointed plotted in Fig. 3. The mean spectrum typically represents spectral neutral of three RGB sub-pixels. Since the sample set comes from a uniform grid design, the mean is expected to be similar to a gray sample (e.g., the spectral reflectance of the white color illustrated in the seventh subplot). 
The eighth subplot highlights the relationship between the percentage variance and eigenvector. The plot indicates that the first three principal directions have the spectral variance of $67 \%$, $22 \%$, and $10 \%$ that is able to contain $99 \%$ of sample data. Hence, these three eigenvectors explain a significant amount of the total variance and the new spectral axes uncover the color information of the sample set. The first eigenvector enlightens the most weighted direction along with samples distributed indicating an overall red cast of the sample set or the reflectance peaks in the long wavelength region. Similarly, the second and the third eigenvectors reveal the variations of the colors in middle and short wavelength regions pointing out the existence of greenish and bluish samples, respectively. If we compare the spectral transmittance of RGB color filters (CF curves show on the first three subplots) with the first three principal directions, the spectral tendencies between them are amazingly consistent while the absolute values exist difference. We acknowledge that the simulated full-color MC EPD is a special case, since its system characteristic matrix is associated with the RGB color filters like a counterpart, whereas the image of LCD is only achieved by the combination of RGB color filters. Clearly, the PCA-based analysis is actually regarded as colorant estimation as Bern's expectation [15], even the colorant-mixture in full-color MC EPD is electrophoretic differentiated from traditional hard copy. As a consequence, the proposed methodology aims to construct a more complicated system such as dual mode switching MC EPD whose inherent physical features are dominated by the interaction of ink particles and colored dyes (RGB or CMY).

The randomly selected 2048 color samples were used to examine the predicting accuracy of the proposed model in MC EPD. Two different sets of tristimulus values, one by sample spectra and the other by the estimated spectra via (8), were applied to calculate the CIEDE2000 color differences for evaluation. If the value is less than one, the difference attributed to predicting error will be indistinguishable for human perception. The color difference values of the whole tested samples were calculated and plotted to build an occurrence chart in Fig. 4. According to the statistic, over $99 \%$ predicting results have reliable accuracy $(\Delta \mathrm{E} 00<1)$ and around $93 \%$ of predictions with high fidelity $(\Delta \mathrm{E} 00<0.5)$. As a result of the verification, the proposed model in Section II can precisely describe the colorimetric performance of MC EPD composed of multi-pigments and color filters.

\section{B. Micro-Encapsulated Type Color EPD}

Of all e-Paper products, micro-encapsulated (ME) EPD manufactured by E Ink has the largest market share due to its excellent and robust performance. In terms of the reliability, a commercial 9-inch full-color ME EPD sample was adopted to confirm the viability of the proposed model. A schematic diagram of the tested sample is illustrated in Fig. 2(b). Unlike the first case whose pixel geometry was RGB side-by-side, full-color ME EPD included four sub-pixels, RGBW (W as white), with quadratic arrangement. Each RGB sub-pixel presented fundamental colorimetric information with 16 intensity levels. The $\mathrm{W}$ channel benefited the enhancement of brightness but sacrificed color fraction simultaneously. A specific algorithm was

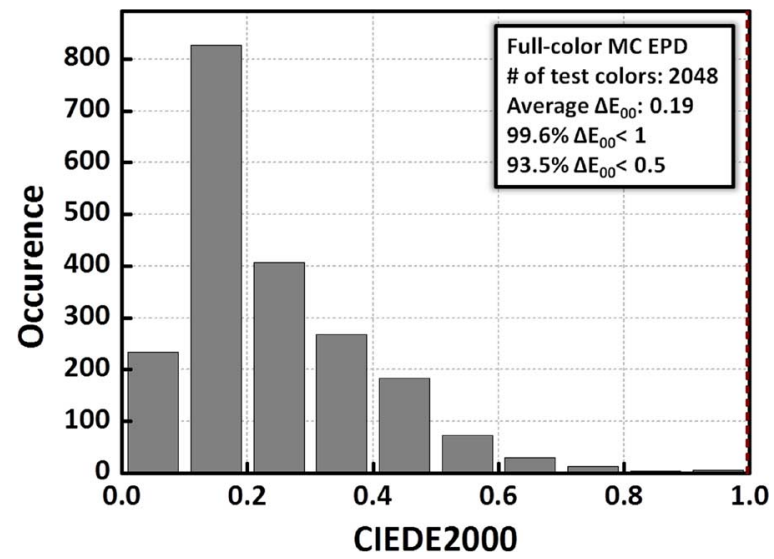

Fig. 4. Occurrence of CIEDE2000 color difference for tested 2048-color samples of simulated full-color MC EPD.

involved to accomplish RGB-to-RGBW input signal-decomposition [26]. Similar change of basis was demonstrated by repeating PCA-based analysis on the spectral reflectance of the identical sample colors used in the first case.

The percentage variance plot, the spectra of the mean and the first six eigenvectors are shown in Fig. 5. Comparing these plots with Fig. 3, the first three principal directions accounted for $99 \%$ of the total variance are no longer the spectral transmittance of color filters. The first eigenvector including $97 \%$ of the representation exhibits a neutral colorant in highly accordance with the spectral reflectance of sample white. It implies that the intensity of reflecting light is a dominant factor mainly resulted from the boosting $\mathrm{W}$ channel in signal controlled algorithm. To have a further discussion, the 1st principal component is regarded as a weighted $\mathrm{DC}$ value attributing to the $\mathrm{W}$ channel plus the mutual portion of the RGB sub-pixels (e.g., for an input signal $(\mathrm{R}, \mathrm{G}, \mathrm{B}, \mathrm{W})=(0.3,0.4,0.5,0.6)$, the DC bias can be considered as $0.6+0.3)$. Except for the significant neutral representation, the variations of chromatic amounts are explained by two complimentary primary spectra (colors) in the next three eigenvectors. For example, the second spectral axis uncovers colors varying along the long wavelength regions (marked as $\mathrm{R}$ ) and the corresponding complementary spectral regions (marked as C) describing the existence of reddish and cyan-ish samples in the data set. Similarly, the last GB primaries are matched up with their MY compliments in the third and forth eigenvectors, respectively. We are aware of that these three principal components are bipolar since the modulated eigen-spectra can either be added to or subtracted from the DC bias. As a consequence, four major axes reveal the momentous directions of the color renderings according to the spectral characteristics of the ME EPD. The linear projections onto the rest of eigenvectors are negligible due to the almost zero contribution of the eigenvalues.

In this case, two sets of sample colors were particularly selected to evaluate the predicting performance of the PCA-based model. One set was made up of a $5 \times 5 \times 5$ grid sampling the entire color-gamut with all the combinations of intensity levels ranked in 3, 6, 9, 12, and 15 (full color-gamut data). The other set included 27 dark colors from all the changes of the least three intensity levels in each RGB channels (dark image data). The measured spectra and tristimulus of both sample sets were introduced to analyze the influence of the numbers of principal 

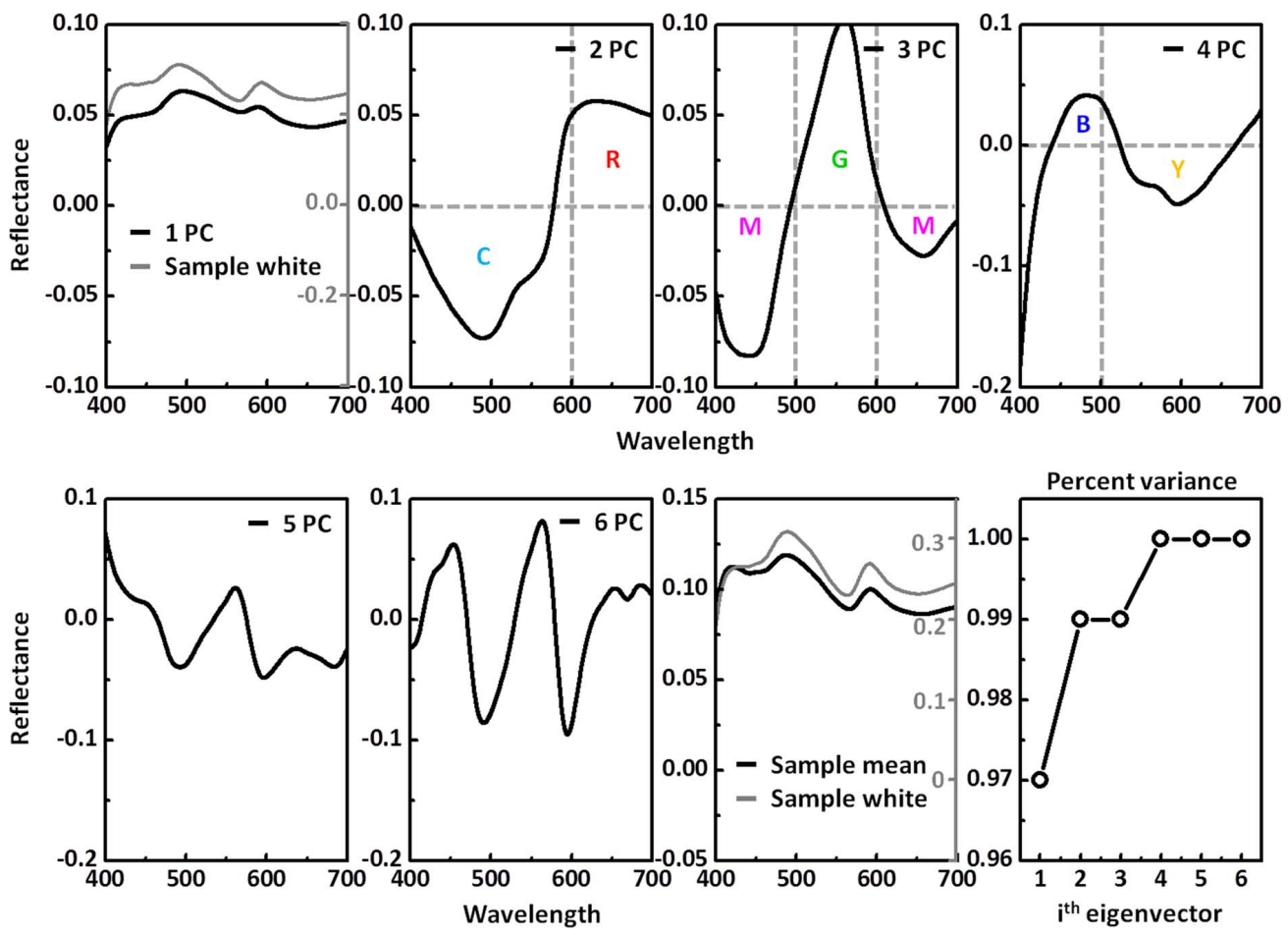

Fig. 5. The first six eigenvectors and the mean derived from the reflectance spectra of the micro-encapsuled EPD sample set. The first eigenvector including $97 \%$ total variance represents the neutral colorant spectrum due to the RGBW pixel layout and driving algorithm. The physical features of chromatic spectra are explained in the second to the forth components by a pair of complimentary primary color.

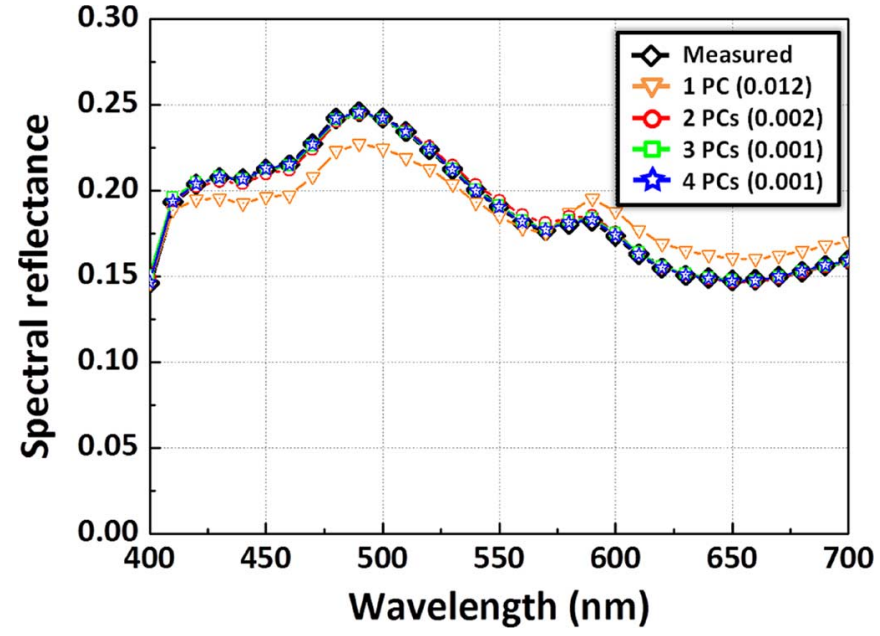

Fig. 6. Comparison of measured spectral reflectance (black line with diamond symbol) with predicted ones from utilizing different numbers of the principal components (from one to four marked with the symbol order of triangle, circle, square and star). The spectral performances evaluated by RMS are listed in the figure as well.

components whose optimal results depend on the spectral and colorimetric accuracy. The spectral accuracy was quantified by the root-mean-square (RMS) error between measurement and estimation. Fig. 6 illustrates one of the measured reflecting spectra and its corresponding estimated spectra with different numbers of eigenvectors. Although one eigenvector is capable of offering $97 \%$ of total variance, the performed spectral fit (marked as triangle symbol, RMS $=0.012$ ) is obviously worse than those applied plural eigenvectors. In other words, the neutral colorant spectrum cannot represent the full result without
TABLE II

Comparison of Colorimetric Performance (CIEDE2000 Color DifFERENCE) AMONG DifFERENT NUMBERS OF PRINCIPAL COMPONENTS FOR Full-Color Micro-CAPSUle EPD PREDicted by PCA-BASED MOdel. Two Sets of Testing Colors Were Involved. One Set Is 125-Colors SpanNing IN Full Color-Gamut of ME EPD, AND the Other Is a Set of 27 COLORS IN DARK IMAGE

\begin{tabular}{cccccccc}
\hline & \multicolumn{3}{c}{ Full color-gamut data } & & \multicolumn{3}{c}{ Dark image data } \\
\cline { 2 - 4 } \cline { 6 - 8 } & Mean & SDV & Max & & Mean & SDV & Max \\
\hline 1 PC & 5.21 & 5.23 & 11.12 & & 7.90 & 0.34 & 9.31 \\
2 PCs & 3.43 & 5.04 & 8.42 & & 7.89 & 0.43 & 9.45 \\
3 PCs & 0.13 & 0.01 & 0.39 & & 0.39 & 0.01 & 0.50 \\
4 PCs & 0.06 & 0 & 0.20 & & 0.19 & 0 & 0.31 \\
\hline
\end{tabular}

any chromatic information. The colorimetric performances specified by CIEDE2000 color difference formula are listed in Table II. The statistic results of predicting accuracies for the full color-gamut data are superior to those of the dark image data. The errors in the end are easily induced when estimating the samples with low input levels due to intrinsically bad reflectance of ME EPD. In addition, employing three or more PCs have even higher colorimetric performance $\left(\Delta \mathrm{E}_{00}<0.4\right)$ than two or less PCs $\left(\Delta \mathrm{E}_{00}>3.4\right)$ despite excellent spectral accuracy for a predicting model with two PCs $(\mathrm{RMS}=0.002)$. The fact also implies that three principal components are sufficient in the ME EPD while the skipped forth eigenvector carries the existence of bluish and yellowish data. As a consequence, the proposed model has been proved to uncover the spectral behavior for the complex full-color ME EPD.

\section{CONCLUSION}

In this work we introduced the principal component analysis to characterize the colorimetric performance by multiple pig- 
ments scenario of an EPD. By factorization of a spectral reflectance set into orthogonal eigenvector basis, we are able to identify the principal directions that a set of reflectance spectra oriented. The first few eigenvectors uncovers the reflectance and scattering features of a set of colorants from their combination. Taking a two-particle type micro-cup EPD with vertical electric field as a case study, the first three eigenvectors closely resemble the spectral transmittance of RGB color filter. In other words, if the profiles of spectral transmittance of color filters can be narrowed and sharpen, the EPD based on this structure will achieve more saturated color. For a case of full-color micro-encapsulated EPD, the first four principal components reveal the correspondingly physical features based on driving algorithm: one neutral colorant spectrum and three chromatic spectra composed of complimentary pairs. Obviously, the EPD utilizing RGBW pixel layout exhibits the weakness of color saturation unless minimizing either the boosting signals or the pixel area of white channel. Subtracting from the mean causes the positive and negative values of the eigenvectors. The spectra modulation about the mean exhibits a ripple effect in higher order eigenvectors.

The PCA-based analysis still leaves many opportunities open and clearly more research must be carried out to explore its potential in full. First of all, all the calculation mentioned here is based on the spectral reflectance. The reason is due to that most available e-Paper displays are monochrome integrated with color filter on top at this moment. As each subpixel is filled with dye fluid or with different color pigment, the discussion of spectral absorptance shall be involved. According to the prior study [27], spectral absorptance is more consistent with linear space for the pigments transposition system. Second, PCA makes a strong assumption that the new (i.e., eigenvector) coordinates are mutually orthogonal. Opponent spectral components are used to estimate the underlying features from the colorant-mixture information. However, neither RGB nor CMY colorant is absolutely orthogonal in spectral reflectance or absorptance. Independent component analysis (ICA) seems to be more adequate in the spectral analysis in color characterization of a composite mixing system.

\section{ACKNOWLEDGMENT}

The authors would like to particularly appreciate P. Yang, J.-M. Hung, and B. Hsieh from SiPix Technology Inc., and S. Huang from E Ink for their technical supports and kind help.

\section{REFERENCES}

[1] I. Ota et al., "Electrophoretic image display (EPID) panel," Proc. IEEE, vol. 61, no., pp. 832-836, 1973.

[2] B. Comiskey et al., "An electrophoretic ink for all-printed reflective electronic displays," Nature, vol. 394, pp. 253-255, 1998.

[3] M. T. Johnson et al., "High-quality images on electrophoretic displays," J. Soc. Inf. Display, vol. 14, p. 175, 2006.

[4] C.-M. Lu et al., "Performance active matrix micro-cup electrophoretic display," in SID Symp. Dig., 2009, vol. 40, p. 1501.

[5] E. Kishi et al., "Development of in-plane EPD," in SID Symp. Dig., 2000, vol. 31, p. 24.

[6] A. R. M. Verschueren et al., "Optical performance of in-plane electrophoretic color e-paper," J. Soc. Inf. Display, vol. 18/1, p. 1, 2010.
[7] N. Hiji et al., "Novel color electrophoretic E-paper using independently movable colored particles," in SID Symp. Dig., 2012, vol. 43, p. 85.

[8] J. Heikenfeld et al., "Electrofluidic displays using Young-Laplace transposition of brilliant pigment dispersions," Nat. Photon., vol. 3, no. 5, pp. 292-296, 2009.

[9] S. Yang et al., "High reflectivity electrofluidic pixels with zero-power grayscale operation," Appl. Phys. Lett., vol. 97, p. 143501, 2010.

[10] J. C. Jones et al., "Novel configuration of the zenithal bistable nematic liquid-crystal device," in SID Symp. Dig., 1998, vol. 29, p. 858.

[11] Y. Asaoka et al., "Polarizer-free reflective LCD combined with ultra low-power driving technology," in SID Symp. Dig., 2009, vol. 40, p. 395.

[12] R. A. Hayes et al., "Video-speed electronic paper based on electrowetting," Nature, vol. 425, no. 6956, pp. 383-385, 2003.

[13] K. Zhou et al., "A full description of a simple and scalable fabrication process for electrowetting displays," J. Micromechan. Microeng., vol. 19, no. 6, pp. 1-12, 2009.

[14] J. Heikenfeld et al., "Review paper: A critical review of the present and future prospects for electronic paper," $J$. SID, vol. 19, no. 2, pp. 129-156, 2011.

[15] R. S. Berns, "A generic approach to color modeling," Color Res. Appl., vol. 22, pp. 318-325, 1997.

[16] J. Heikenfeld, "A new bi-primary color system for doubling the reflectance and colorfulness of e-paper," in Proc. SPIE, 2011, Advances in Display Technologies; and E-papers and Flexible Displays, p. 795608.

[17] H. R. Kang, Color Technology for Electronic Imaging Devices. Bellingham, WA: SPIE Press, 1996.

[18] F. H. Imai et al., "Principal component analysis of skin color and its application to colorimetric color reproduction on CRT display and hardcopy,” J. Imag. Sci. Technol., vol. 40, pp. 422-430, 1998.

[19] D.-Y. Tzeng et al., "A review of principal component analysis and its applications to color technology," Color Res. Appl., vol. 30, pp. 84-89, 2005.

[20] Q. Du et al., "Low-complexity principal component analysis for hyperspectral image compression," Int. J. High Performance Comput. Appl., vol. 22 , no. 4, pp. 438-448, 2008.

[21] K. Pearson, "On lines and planes of closest fit to systems of points in space," Phil. Mag. A, vol. 6, pp. 559-572, 1901.

[22] I. T. Jolliffe, Principal Component Analysis, 2nd ed. New York, NY, USA: Springer, 2002.

[23] B. Moore, "Principal component analysis in linear systems: Controllability, observability, and model reduction," IEEE Trans. Autom. Control, vol. AC-26, pp. 17-32, 1981.

[24] G. Strang, Introduction to Linear Algebra, 4th ed. Wellesley, MA, USA: Wellesley Cambridge, 2009.

[25] P. Green et al., Colour Engineering-Achieving Device Independent Colour. Chichester, U.K.: Wiley, 2002.

[26] Y. Kwak et al., "Generating vivid colors on red-green-blue-white electonic-paper display," Appl. Opt., vol. 47, pp. 4491-4500, 2008.

[27] Y.-H. Lu et al., "Colorimetric characterization of monochromatic micro-cup electrophoretic display," in SID Symp. Dig., 2011, vol. 42.

Yen-Hsing Lu received the B.S. degree in communication engineering from National Chiao Tung University, Taiwan in 2004. He is currently a Ph.D. candidate in the Institute of Electro-Optical Engineering at National Chiao Tung University. His current researches are the colorimetric characterization for novel electronic printing device.

Chung-Hao Tien received the B.S. degree in communication-engineering and the Ph.D. degree in electro-optical engineering from National Chiao Tung University (NCTU), Hsinchu, Taiwan, in 1997 and 2003, respectively.

After working as a research assistant at University of Arizona (2001) and a Post-Doctoral Fellow at Carnegie Mellon University (2003), he joined NCTU as an assistant professor in the Department of Photonics. His research work was in the area of computational imaging, free-form optics, display, and lighting optics.

Dr. Tien is a member of the Phi Tau Phi Honor Society, OSA, SPIE, and SID. 